\title{
Evaluating CT Perfusion Deficits in Global Cerebral Edema after Aneurysmal Subarachnoid Hemorrhage
}

\author{
H. Baradaran, V. Fodera, D. Mir, K. Kesavobhotla, J. Ivanidze, U. Ozbek, A. Gupta, J. Claassen, and P.C. Sanelli
}

\begin{abstract}
BACKGROUND AND PURPOSE: Global cerebral edema is an independent predictor of mortality and poor outcomes after aneurysmal $\mathrm{SAH}$. Global cerebral edema, a complex disease process, is thought to be associated with an altered cerebral autoregulatory response. We studied the association between cerebral hemodynamics and early global cerebral edema by using CTP.
\end{abstract}

MATERIALS AND METHODS: We retrospectively studied consecutive patients with aneurysmal SAH with admission CTP performed at days 0 -3. Two neuroradiologists classified global cerebral edema and hydrocephalus on NCCT performed concurrently with CTP. Global cerebral edema was defined as diffuse effacement of the sulci and/or basal cisterns or diffuse disruption of the cerebral gray-white matter junction. CTP was postprocessed into CBF and MTT maps by using a standardized method. Quantitative analysis of CTP was performed by using standard protocol with ROI sampling of the cerebral cortex. The Fisher exact test, Mann-Whitney test, and independent-samples $t$ test were used to determine statistical associations.

RESULTS: Of the 45 patients included, $42 \%$ (19/45) had global cerebral edema and 58\% (26/45) did not. Patient groups with and without global cerebral edema were well-matched for demographic and clinical data. Patients with global cerebral edema were more likely to have qualitative global CTP deficits than those without global cerebral edema $(P=.001)$ with an OR $=13.3(95 \% \mathrm{Cl}, 2.09-138.63)$. Patients with global cerebral edema also had a very strong trend toward statistical significance, with reduced quantitative CBF compared with patients without global cerebral edema $(P=.064)$.

CONCLUSIONS: Global perfusion deficits are significantly associated with global cerebral edema in the early phase after aneurysmal SAH, supporting the theory that hemodynamic disturbances occur in global cerebral edema.

ABBREVIATIONS: $G C E=$ global cerebral edema; $\mathrm{aSAH}=$ aneurysmal subarachnoid hemorrhage; $\mathrm{DCl}=$ delayed cerebral ischemia

A neurysmal subarachnoid hemorrhage (aSAH) is a devastating disease with serious complications and lasting impairment in patients who survive. It has a mortality rate ranging from

Received September 3, 2014; accepted after revision January 18, 2015.

From the Departments of Radiology (H.B., V.F., D.M., K.K., J.I., A.G.) and Public Health (U.O.), NewYork-Presbyterian Hospital, Weill Cornell Medical College, New York, New York; Department of Neurology (J.C.), NewYork-Presbyterian Hospital, Columbia University Medical Center, New York, New York; and Department of Radiology (P.C.S.), North-Shore-Long Island Jewish Health System, Great Neck, New York.

This work was supported by Grant 5K23NS058387 from the National Institute of Neurological Disorders and Stroke, a component of the National Institutes of Health.

Paper previously presented at: Annual Meeting of the American Society of Neuroradiology and the Foundation of the ASNR Symposium, May 18-23, 2013; San Diego, California.

Please address correspondence to H. Baradaran, MD, Department of Radiology, NewYork-Presbyterian Hospital, Weill Cornell Medical College, 525 East 68th St Starr 8A, NY, NY 10065; e-mail: heb2017@nyp.org

- Indicates open access to non-subscribers at www.ajnr.org

http://dx.doi.org/10.3174/ajnr.A4328
$32 \%$ to $67 \%{ }^{1-3}$ and accounts for up to $7 \%$ of all strokes. ${ }^{4}$ While there has been some minimal improvement in the mortality rate since the 1960s, the persistently poor outcomes make aSAH a serious disease. Poor outcomes occur after survival from the initial aneurysm rupture, with long-term functional disability in more than half of patients, of whom $26 \%$ have persistent dependence. ${ }^{5}$ Additionally, as many as $20 \%$ of patients have global cognitive impairment contributing to poor functional status. ${ }^{6}$ Thus, aSAH is associated with a substantial burden on health care resources, most of which are related to long-term care for functional and cognitive disability. ${ }^{7}$

After aneurysm rupture, early global cerebral edema (GCE) contributes significantly to functional and cognitive disability as a secondary complication. ${ }^{7-12}$ GCE typically occurs in the early phase (days $0-3$ ) after $\mathrm{SAH}^{10-12}$ and has been shown to be an independent predictor of morbidity and mortality. ${ }^{10}$ Currently, detection of GCE is limited to qualitative assessment of subtle findings on NCCT. ${ }^{10}$ While this finding is fairly common, it is 
often difficult to appreciate and quantify, so the true incidence of GCE is likely underreported. ${ }^{10}$ The lack of quantitative markers is a major limitation for accurate detection and monitoring of GCE to guide management. Although little is known about the mechanisms underlying GCE, microvascular dysfunction from diffuse ischemic injury has been implicated in the initial circulatory arrest at the time of aneurysm rupture. ${ }^{10}$ The neurotoxic effects from the breakdown of blood products after the initial circulatory arrest may lead to hemodynamic disturbances in autoregulatory response and neuronal dysfunction, ${ }^{10}$ which may be involved in the pathophysiology of GCE. ${ }^{13-16}$

GCE is a complex and poorly understood disease process with many contributing factors. Understanding the mechanisms underpinning the development of GCE can aid in prevention and treatment of this disease process. Because of the potential role of hemodynamic disturbance in GCE, we evaluated cerebral perfusion parameters, including $\mathrm{CBF}$ and MTT by using CTP in patients with early GCE after aSAH.

\section{MATERIALS AND METHODS Study Population}

This was an institutional review board-approved retrospective study of consecutive patients with aSAH with CTP examinations performed on admission (days 0-3) between October 2008 to March 2011 at New York-Presbyterian Hospital-Weill Cornell. A total of 105 patients were admitted with aneurysmal subarachnoid hemorrhage; however, patients were only included if they had a baseline CTP examination on days $0-3$ of admission according to our standard scanning protocol. The presence of GCE and the degree of hydrocephalus were determined on NCCT performed concurrently with CTP by 2 neuroradiologists by consensus. They interpreted the NCCT and CTP findings at separate time points, blinded to all other clinical and imaging data. We used a 2-part definition for GCE that was similar to that in published criteria: 1) complete or near-complete effacement of the hemispheric sulci and basal cisterns, and 2) bilateral and extensive disruption of the cerebral gray-white matter junction at the level of the centrum semiovale. ${ }^{10}$ Patients were classified as having GCE if either diffuse effacement of the sulci and basal cisterns or diffuse disruption of the cerebral gray-white matter junction was present on the admission NCCT scan. Hydrocephalus was classified as none, mild, moderate, or severe by 2 neuroradiologists by consensus on the basis of the admission NCCT. All demographic and clinical data, including Glasgow Coma Scale scores, Hunt and Hess scores, smoking history, and history of hypertension, were obtained from retrospective chart review.

\section{CTP Scanning, Postprocessing, and Data Collection}

CTP was performed on admission (days 0-3) in all patients with aSAH before aneurysm treatment. We performed initial noncontrast CT of the head from the foramen magnum to the vertex, using 5.0-mm-thick sections with $120 \mathrm{kV}$ (peak), $250 \mathrm{mAs}$, and 1.0 rotation time. A standard scanning protocol for CTP at our institution uses the HD750 scanner (GE Healthcare, Milwaukee, Wisconsin) with a cine $4 \mathrm{i}$ scanning mode and a 45-second acquisition at 1 rotation per second with $80 \mathrm{kVp}$ and $190 \mathrm{~mA}$. A scanning volume of $2.0 \mathrm{~cm}$ was used, consisting of 4 sections at $5.0-\mathrm{mm}$ thickness with its inferior extent selected at the level of the basal ganglia, above the orbits, to minimize radiation exposure to the lenses. Approximately $45 \mathrm{~mL}$ of nonionic iodinated contrast was administered intravenously at $5 \mathrm{~mL} / \mathrm{s}$ by using a power injector with a 5 -second delay.

Postprocessing of the acquired images into CBF, MTT, and CBV maps was performed on an Advantage Workstation by using CTP software, Version 3.0 (GE Healthcare). This software uses a deconvolution method, which is considered most accurate for low-contrast injection rates. ${ }^{17}$ The postprocessing technique was standardized for all patients according to recommended guidelines, ${ }^{18}$ with the arterial input function as the A2 segment of the anterior cerebral artery ${ }^{19}$ and the venous function as the superior sagittal sinus. In the deconvolution method, selection of the arterial input function has been shown to not significantly affect the quantitative perfusion values. ${ }^{18,20,21}$

The perfusion maps were qualitatively evaluated by 2 neuroradiologists (with 7 and 10 years' experience) blinded to clinical and imaging data, to determine the presence of global perfusion deficits, defined as diffusely decreased CBF and/or elevated MTT. Focal perfusion abnormalities due to the primary hemorrhagic event, surgical intervention, hematoma, or vasospasm as identified on the acquired images from the CTP dataset were not included as perfusion deficits related to early global cerebral ischemia. After the images were reviewed independently, consensus judgment was determined.

Quantitative analysis was conducted by using a standardized method with contiguous ROI placement, measuring $157 \mathrm{~mm}^{2}$, sampling the cerebral cortex. Each CTP section had up to 24 ROIs distributed along the cortical surfaces to include mostly gray matter in the following territories: approximately 6 ROIs in the anterior cerebral artery, 12 in the MCA, and 6 in the posterior cerebral artery (Fig 1). CTP studies were analyzed with readers blinded to all clinical and imaging data to limit test-review bias.

\section{Statistical Analysis}

The incidence of qualitative diffuse CTP deficits was calculated for each outcome group (GCE and non-GCE). Two-tailed $P$ values were calculated by using the Fisher exact test to determine statistical significance. The OR was also calculated to determine the strength of association between GCE and CTP deficits. The Mann-Whitney test was used to determine the statistical difference between patient groups for demographic characteristics.

Quantitative CTP data were analyzed by calculating the mean CBF, CBV, and MTT and their SDs for each outcome group by using all ROIs for all 4 section locations included in the arithmetic means. To minimize the contribution of vascular pixels from large vessels, we excluded CBF values of $>100 \mathrm{~mL} / 100 \mathrm{~g} / \mathrm{min}$ from the statistical analysis and did not use these in calculating the mean CBF, CBV, and MTT because this method has been published in the evaluation of ischemia. ${ }^{22}$ The ROIs in the perfusion abnormalities due to the primary hemorrhagic event and/or surgical intervention were not included in the statistical analysis. A Student 2-tailed $t$ test was used to determine statistical significance, accepted at $P<.05$.

Interobserver reliability for the detection of GCE between the 2 neuroradiologists was calculated by using the Cohen $\kappa$ statistic 


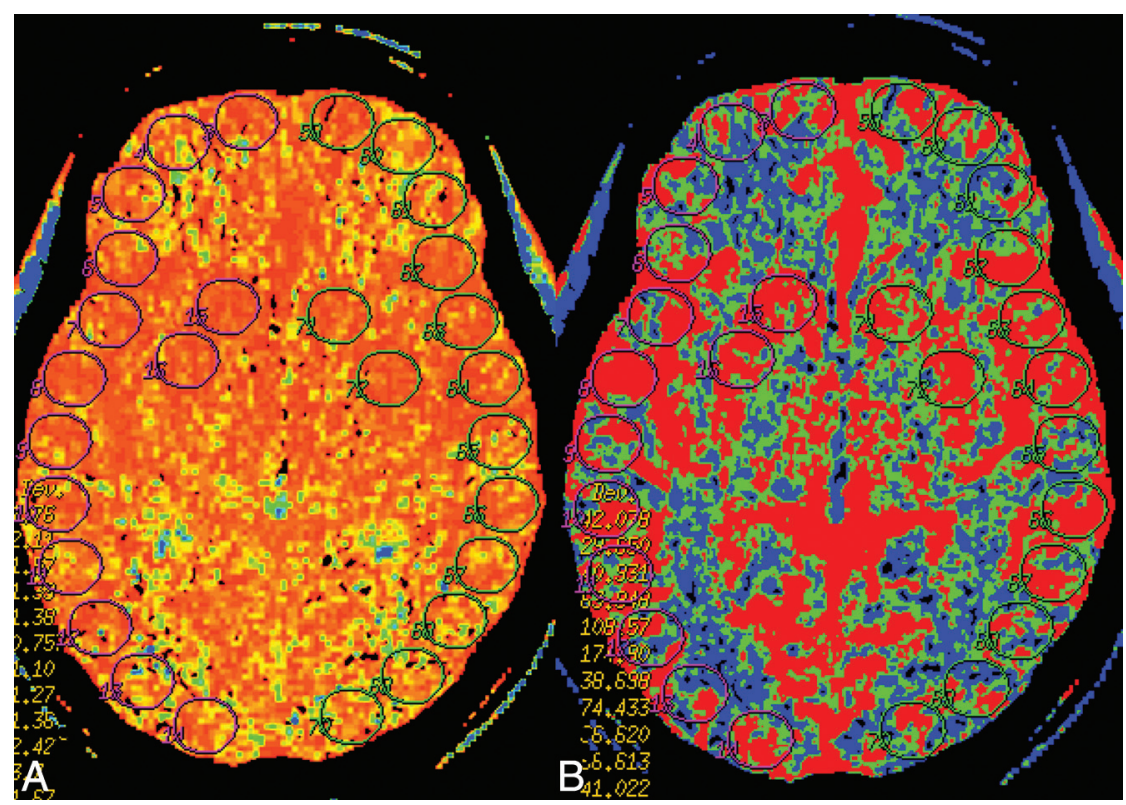

FIG 1. Example of ROI placement on the MTT $(A)$ and $C B F(B)$ maps in a representative patient.

Demographic, clinical, and imaging characteristics ${ }^{a}$

\begin{tabular}{lccc}
\hline & No GCE & GCE & $\begin{array}{c}\text { Statistical } \\
\text { Comparison }\end{array}$ \\
\hline Age (yr) & $55.4 \pm 13.5$ & $49.7 \pm 12.56$ & $Z=0.36$ \\
Sex & $76.9 \%(\mathrm{female})$ & $73.7 \%(\mathrm{female})$ & $P=1$ \\
Smoking history & $50 \%(13 / 26)$ & $42 \%(8 / 19)$ & $P=.76$ \\
History of hypertension & $42 \%(11 / 26)$ & $63 \%(12 / 19)$ & $P=.23$ \\
Admission Hunt and Hess score & $2.58 \pm 1.00$ & $2.95 \pm 1.10$ & $P=.64$ \\
Admission GCS & $13-14$ & $13-14$ & $P=.67$ \\
Hydrocephalus & $1.77 \pm 0.80$ (mild) & $2.00 \pm 0.65$ (mild) & $P=.368$ \\
Global CBF & $40.3 \pm 11.0$ & $34.8 \pm 7.6$ & $P=.06$ \\
Global MTT & $5.6 \pm 1.56$ & $6.38 \pm 2.02$ & $P=.15$ \\
\hline
\end{tabular}

Note:-GCS indicates Glasgow Coma Scale.

${ }^{a}$ Mean values are given for age, admission Hunt and Hess score, admission GCS, hydrocephalus, global CBF, and global MTT.

with separate analyses for each criterion of sulcal effacement and gray-white matter differentiation.

\section{RESULTS}

Forty-five patients with aSAH and admission CTP studies were included in the statistical analysis. Of these 45 patients, $42.2 \%$ (19/45) were classified as having GCE, and 57.8\% (26/45), without GCE. Patient groups with and without GCE were wellmatched for basic demographic characteristics including age, sex, admission Hunt and Hess and Glasgow Coma Scale scores, and hydrocephalus (Table). Qualitative global perfusion deficits were seen in $53 \%(10 / 19)$ of patients with GCE and $7.7 \%(2 / 26)$ of patients without GCE. No evidence of vasospasm was seen on either the CTA or CTP examinations for the patients included in the study. The Fisher exact test revealed that patients with GCE had significantly higher rates of global perfusion deficits $(P=$ .001) compared with patients without GCE (Fig 2) with an OR = 13.3 (95\% CI, 2.09-138.63). The mean global CBF in patients without GCE was $40.31 \mathrm{~mL} / 100 \mathrm{~g} / \mathrm{min}$ compared with $34.75 \mathrm{~mL} /$ $100 \mathrm{~g} / \mathrm{min}$ in patients with GCE $(P=.064)$. The mean global MTT in patients with GCE was 6.38 seconds compared with $5.60 \mathrm{sec}-$ onds in patients without GCE $(P=.153)$. The mean global CBV in patients with GCE was $2.29 \mathrm{~mL} / 100 \mathrm{~g}$ compared with $2.16 \mathrm{~mL} / 100 \mathrm{~g}$ in patients without GCE $(P=.78)$. The Cohen $\kappa$ statistic for interobserver reliability for classification of sulcal effacement was high, with a value of $0.86(P<.0001)$, and the Cohen $\kappa$ statistic for classification of gray-white matter differentiation showed a medium association, with a value of $0.73(P<.0001)$.

\section{DISCUSSION}

GCE is fairly commonly encountered after aSAH and has been shown to have associated devastating consequences. ${ }^{10}$ The presence of GCE on initial head NCCT is an independent predictor of poor outcome and mortality. ${ }^{10}$ Despite its relatively common occurrence, GCE is an incompletely understood, complex multifactorial process with several proposed contributing etiologies including metabolic disturbances, increased intracranial pressure, and global ischemia. ${ }^{13,15}$ Additionally, GCE is associated with breakdown of the blood-brain barrier, leading to disturbances in the autoregulatory response, which may manifest as hemodynamic alterations in perfusion. ${ }^{13,14}$

Our results support global perfusion deficits on CTP occurring more frequently in patients with GCE compared with patients without GCE. Furthermore, the patient groups with and without GCE did not have significant differences in key demographic features, such as age and sex or history of smoking or hypertension. Increased disease severity cannot be attributed to increased perfusion deficits because our patients were also well-matched for measures of disease severity, including Glasgow Coma Scale scores and Hunt and Hess scores. Additionally, even though hydrocephalus has been shown to cause global perfusion deficits ${ }^{23}$ and could potentially confound our analysis, most important, we found no statistical difference in the severity of hydrocephalus between the GCE and non-GCE groups. Also, although vasospasm is a common complication of aSAH and could potentially confound perfusion deficits, our patients were imaged within the first 3 days after initial presentation when vasospasm is less likely to occur. Typically, development of vasospasm after aSAH is thought to peak at 7 days. ${ }^{24,25}$ Additionally, focal perfusion deficits of any kind were excluded, and only global deficits were considered true perfusion deficits suggestive of global cerebral edema.

The exact mechanism underlying GCE and its role in patients with aSAH developing serious neurologic complications are not clearly understood. Some studies have suggested that the initial bleeding insult causes high intracranial pressure leading to 


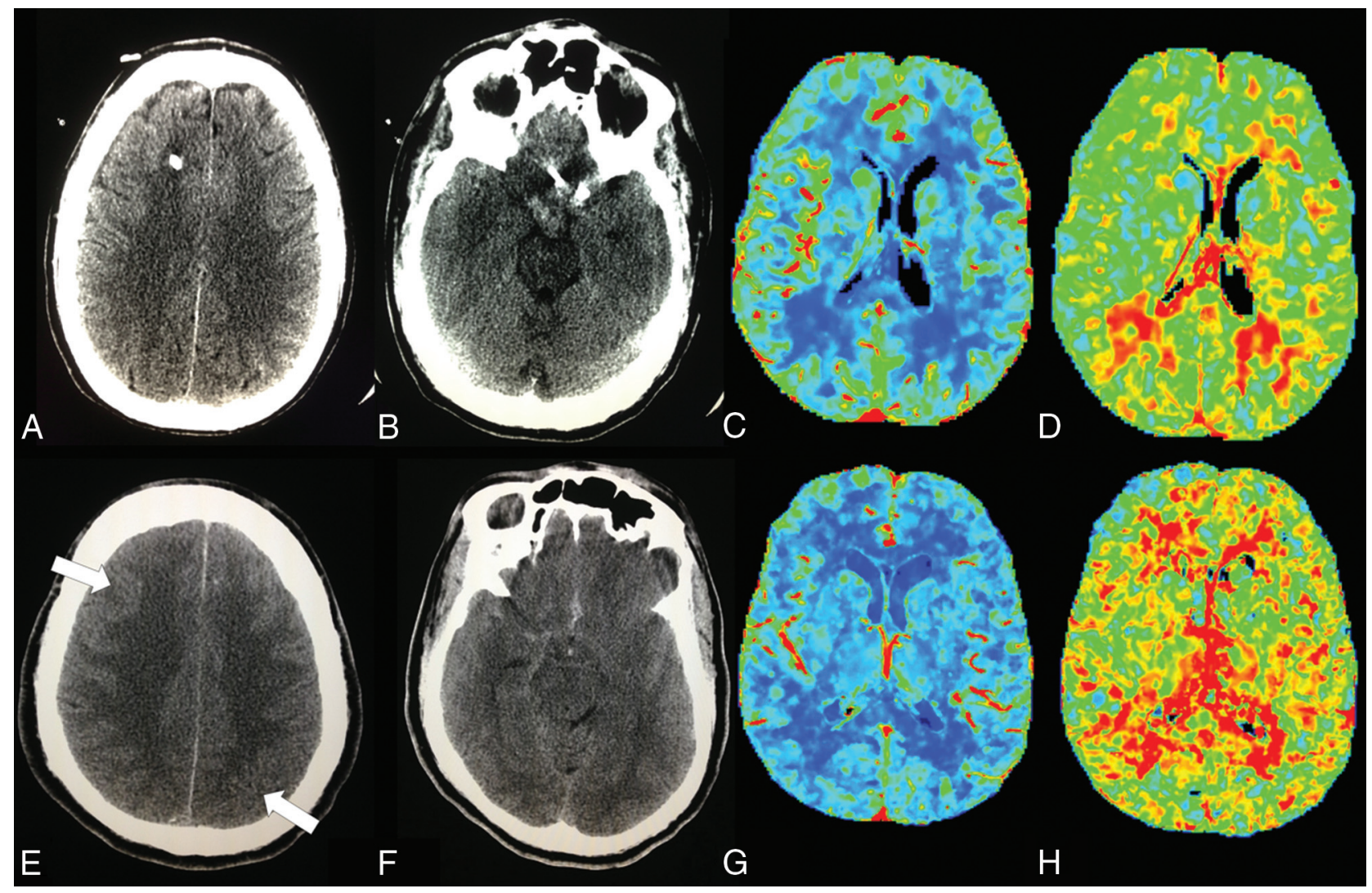

FIG 2. Patient 1 is an 87 -year-old woman with acute aSAH from a right posterior communicating artery aneurysm who presented with Hunt and Hess grade 4 and a Glasgow Coma Scale score of 7-12. No GCE is seen on NCCT $(A$ and $B)$. Normal findings on CBF $(C)$ and MTT (D) maps are seen in this patient. Patient 2 is a 53-year-old man with right middle cerebral artery aneurysmal rupture who presented with Hunt and Hess grade 2 and a Glasgow Coma Scale score of 15. GCE is seen on NCCT with a loss of gray-white differentiation at the level of the centrum semiovale as demonstrated by fingerlike projections of white matter extending to the cortex ${ }^{10}(E$, arrows) and diffuse effacement of the basal cisterns and cerebral sulci $(F)$. Globally decreased $\operatorname{CBF}(G)$ and increased MTT $(H)$ are seen in this patient.

cerebral circulatory arrest causing ischemic brain injury. This may lead to cytotoxic edema and breakdown of the blood-brain barrier with disturbances in the autoregulatory response from inflammatory and neurotoxic effects on the vasomotor centers of the brain. ${ }^{13,14}$ Recently, microarterial constriction and microthrombosis have been shown to contribute to the early damage leading to delayed cerebral ischemia (DCI). ${ }^{26}$ Although the relationship between the vascular pathology in DCI and GCE is not well-understand, microvascular dysfunction represents a plausible mechanistic link between DCI and GCE, which warrants further investigation.

Our study demonstrates that patients with GCE are more likely to have global perfusion deficits with reduced CBF and/or elevated MTT compared with patients without GCE, confirming that disturbances in the autoregulatory response occur in patients with GCE. Further investigation is needed to fully elucidate the underlying pathophysiologic mechanism leading to GCE.

Our study has some limitations. First, our sample size was relatively small, and though our results achieved statistical significance, the wide confidence interval in our OR suggests that additional work is needed to more precisely determine the strength of the association of global perfusion deficits with GCE. Second, the diagnosis of GCE on NCCT can be prone to subjective assessment. The proportion of patients with GCE in our aSAH cohort was $42.2 \%$, which is in the $6 \%-62 \%$ range of previously published studies. ${ }^{10,15,27}$ Additionally, we had medium-to-high interobserver reliability between our neuroradiologists for the 2-part definition of global cerebral edema used. While the interobserver reliability was high for sulcal effacement, indicating that it may be a more important factor in the definition of GCE than loss of gray-white differentiation, other imaging markers, such as perfusion metrics, might aid in the definition of GCE. Although our study was adequately powered to assess our primary outcome of qualitative global perfusion deficits, we were not able to demonstrate the statistical significance in quantitative CBF between the 2 groups, despite a strong trend. Further work should be done with larger sample sizes to evaluate the quantitative assessment of CBF.

In summary, our study reveals that patients with aSAH with GCE have a statistically increased incidence of global perfusion deficits on CTP in the early phase (days 0-3) compared with those without GCE, supporting the theory that GCE is associated with altered cerebral hemodynamics. However, further work is needed to understand the pathologic mechanisms leading to altered hemodynamics in GCE and to assess the clinical implications of global perfusion deficits in contributing to poor outcomes after aSAH.

\section{CONCLUSIONS}

Patients with aSAH who have GCE on early-phase (days 0-3) NCCT are significantly more likely to have global perfusion deficits on CTP compared with patients without GCE. GCE and its 
relationship with poor clinical outcomes in patients with aSAH are complex, but our study supports the theory that GCE is associated with altered cerebral hemodynamics.

Disclosures: Jana Ivanidze — UNRELATED: Grants/Grants Pending: Radiological Society of North America, ${ }^{*}$ Comments: Radiological Society of North America Resident Research Grant. Umut Ozbek_UNRELATED: Grants/Grants Pending: Clinical and Translational Science Center at Weill Cornell Medical College (UL1TR000457-06); Ajay Gupta-UNRELATED: Grants/Grants Pending: Foundation of the American Society of Neuroradiology Scholar Award, ${ }^{*}$ Association of University Radiologists GE Radiology Research Academic Fellowship Grant.* Jan Claassen-UNRELATED: Board Membership: Actelion (Advisory Board).* *Money paid to the institution.

\section{REFERENCES}

1. Hop JW, Rinkel GJ, Algra A, et al. Case-fatality rates and functional outcome after subarachnoid hemorrhage: a systematic review. Stroke 1997;28:660-64

2. Ingall T, Asplund $\mathrm{K}$, Mähönen M, et al. Project for the WHOM: a multinational comparison of subarachnoid hemorrhage epidemiology in the WHO MONICA stroke study. Stroke 2000;31:1054-61

3. Longstreth WT, Nelson LM, Koepsell TD, et al. Clinical course of spontaneous subarachnoid hemorrhage: a population-based study in King County, Washington. Neurology 1993;43:712

4. Feigin VL, Lawes CM, Bennett DA, et al. Stroke epidemiology: a review of population-based studies of incidence, prevalence, and case-fatality in the late 20th century. Lancet Neurol 2003;2:43-53

5. Molyneux AJ, Kerr RSC, Birks J, et al. Risk of recurrent subarachnoid haemorrhage, death, or dependence and standardised mortality ratios after clipping or coiling of an intracranial aneurysm in the International Subarachnoid Aneurysm Trial (ISAT): long-term follow-up. Lancet Neurol 2009;8:427-33

6. Springer MV, Schmidt JM, Wartenberg KE, et al. Predictors of global cognitive impairment 1 year after subarachnoid hemorrhage. $\mathrm{Neu}$ rosurgery 2009;65:1041-43

7. Roos YB, Dijkgraaf MG, Albrecht KW, et al. Direct costs of modern treatment of aneurysmal subarachnoid hemorrhage in the first year after diagnosis. Stroke 2002;33:1595-99

8. Mayer SA, Kreiter KT, Copeland D, et al. Global and domain-specific cognitive impairment and outcome after subarachnoid hemorrhage. Neurology 2002;59:1750-58

9. Hackett ML, Anderson CS. Health outcomes 1 year after subarachnoid hemorrhage: an international population-based study-the Australian Cooperative Research on Subarachnoid Hemorrhage Study Group. Neurology 2000;55:658-62

10. Claassen J, Carhuapoma JR, Kreiter KT, et al. Global cerebral edema after subarachnoid hemorrhage: frequency, predictors, and impact on outcome. Stroke 2002;33:1225-32

11. Kreiter KT, Copeland D, Bernardini GL, et al. Predictors of cognitive dysfunction after subarachnoid hemorrhage. Stroke 2002; 33:200-08

12. Lagares A, Gómez PA, Lobato RD, et al. Prognostic factors on hos- pital admission after spontaneous subarachnoid haemorrhage. Acta Neurochir (Wien) 2001;143:665-72

13. Mocco J, Prickett CS, Komotar RJ, et al. Potential mechanisms and clinical significance of global cerebral edema following aneurysmal subarachnoid hemorrhage. Neurosurg Focus 2007;22:E7

14. Zetterling M, Hallberg L, Hillered L, et al. Brain energy metabolism in patients with spontaneous subarachnoid hemorrhage and global cerebral edema. Neurosurgery 2010;66:1102-10

15. Helbok R, Ko SB, Schmidt JM, et al. Global cerebral edema and brain metabolism after subarachnoid hemorrhage. Stroke 2011; 42:1534-39

16. Grote E, Hassler W. The critical first minutes after subarachnoid hemorrhage. Neurosurgery 1988;22: 654-61

17. Wintermark M, Maeder P, Thiran JP, et al. Quantitative assessment of regional cerebral blood flows by perfusion CT studies at low injection rates: a critical review of the underlying theoretical models. Eur Radiol 2001;11:1220-30

18. Sanelli PC, Lev MH, Eastwood JD, et al. The effect of varying userselected input parameters on quantitative values in CT perfusion maps. Acad Radiol 2004;11:1085-92

19. Wintermark M, Lau BC, Chien J, et al. The anterior cerebral artery is an appropriate arterial input function for perfusion-CT processing in patients with acute stroke. Neuroradiology 2008;50:227-36

20. Shin BJ, Anumula N, Hurtado-Rúa S, et al. Does the location of the arterial input function affect quantitative $C T P$ in patients with vasospasm? AJNR Am J Neuroradiol 2014;35:49-54

21. Ferreira RM, Lev MH, Goldmakher G V, et al. Arterial input function placement for accurate CT perfusion map construction in acute stroke. AJR Am J Roentgenol 2010;194:1330-36

22. Murphy BD, Fox AJ, Lee DH, et al. Identification of penumbra and infarct in acute ischemic stroke using computed tomography perfusion-derived blood flow and blood volume measurements. Stroke 2006;37:1771-77

23. van Asch CJ, van der Schaaf IC, Rinkel GJ. Acute hydrocephalus and cerebral perfusion after aneurysmal subarachnoid hemorrhage. AJNR Am J Neuroradiol 2010;31:67-70

24. Aralasmak A, Akyuz M, Ozkaynak C, et al. CT angiography and perfusion imaging in patients with subarachnoid hemorrhage: correlation of vasospasm to perfusion abnormality. Neuroradiology 2009;51:85-93

25. Weidauer S, Lanfermann H, Raabe A, et al. Impairment of cerebral perfusion and infarct patterns attributable to vasospasm after aneurysmal subarachnoid hemorrhage: a prospective MRI and DSA study. Stroke 2007;38:1831-36

26. Vergouwen MD, Vermeulen M, Coert BA, et al. Microthrombosis after aneurysmal subarachnoid hemorrhage: an additional explanation for delayed cerebral ischemia. J Cereb Blood Flow Metab 2008;28:1761-70

27. Kassell NF, Torner JC, Haley EC, et al. The International Cooperative Study on the Timing of Aneurysm surgery. Part 1: overall management results. J Neurosurg 1990;73:18-36 
n the author listing for the article "Evaluating CT Perfusion Deficits in Global Cerebral Edema after Aneurysmal Subarachnoid

Hemorrhage” (AJNR Am J Neuroradiol 2015;36:1431-35, originally published on-line on May 14, 2015, doi:10.3174/ajnr.A4328) the name of the fourth author was spelled incorrectly. The correct author list appears below. The Journal regrets this error.

Baradaran H, Fodera V, Mir D, Kesavabhotla K, Ivanidze J, Ozbek U, Gupta A, Claassen J, Sanelli PC.

http://dx.doi.org/10.3174/ajnr.A4565 